East African Medical Journal vol. 77 No. 2 February 2000

GENERATIONAL DIFFERENCES IN MALE SEXUALITY THAT MAY AFFECT ZIMBABWEAN WOMEN'S RISK FOR SEXUALLY TRANSMITTED DISEASES AND HIV/AIDS

B.A. Olayinka, M.S, UCLA Drug Abuse Research Center, Los Angeles, California, USA, L. Alexander, BSC, School of Public Health, University of Alabama, Birmingham Alabama, USA, M.T. Mbizvo, Ph.D Human Reproduction Programme, World Health Organization, Geneva, Switzerland and L. Gibney. Ph.D, School of Public Health, University of Alabama, Birmingham Alabama, USA.

Request for reprints to: B.A. Olayinka, UCLA Drug Abuse Research Center, 1640 S. Sepulveda Blvd., Suite 200, Los Angeles, CA 90025 , USA

\title{
GENERATIONAL DIFFERENCES IN MALE SEXUALITY THAT MAY AFFECT ZIMBABWEAN WOMEN'S RISK FOR SEXUALLY TRANSMITTED DISEASES AND HIV/AIDS
}

\author{
B.A. OLAYINKA, L. ALEXANDER, M.T. MBIZVO and L. GIBNEY
}

\begin{abstract}
Objective: To determine generational differences in male sexuality, which could predispose men's female sexual partners to STDs/HIV.

Design: Cross-sectional study.

Setting: Harare, Zimbabwe.

Subjects: Three hundred and ninety seven male adults aged eighteen years and above.

Main outcome measures: Number of sexual partners, condom use, likelihood of sharing information on HIV status with wife or with other sexual partners, preference for women with dry vaginas prior to sex and discussion about sexual satisfaction with wife or with girl friend.

Results: Fewer men in the 27-39 year age group when compared to men aged 18-26 years $\mathbf{2 2 . 2 \%}$ versus $\mathbf{2 8 . 9 \%}$ ) had two or more sexual partners. The greatest proportion of ever condom users were men aged 27-39 years, but this proportion was not significantly different from the proportion of ever condom users aged $18-26$ years $(76.5 \%$ versus $69.5 \%$; Odds Ratio $[O R]=1.42,95 \%$ Confidence interval $[C I]$ 0.81-2.51). Men aged $\geq 40$ years $(43.8 \%$, OR $=0.34,95 \% \mathrm{CI} 0.20-0.84)$ were significantly fewer than men aged 18-26 years in ever use of condoms. In the event that they contracted HIV, 79.5\%, 82.4\% and 85.9\% of men aged 1826, 27-39 and 40 years and above respectively indicated that they would disclose their HIV status to their wives. On the other hand, men aged 18-26 years $(\mathbf{5 6 . 8 \%}), 27-39$ years $(\mathbf{5 4 . 0 \%})$ and $\geq \mathbf{4 0}$ years $(53.1 \%)$ indicated that they would disclose their HIV status to girl friends or other sexual partners. Significantly, more men aged 40 years $(O R=2.23$; 95\% CI 1.19-4.18) and 27-39 years $(\mathrm{OR}=1.82 ; 95 \%$ CI 1.00-3.32) in comparison to 18-26 year old men indicated their preference for women with dry vaginas prior to sex. The greatest proportion of men who discussed sexual satisfaction with their wives $(85.7 \%)$ and girl friends or other sexual partners $(23.5 \%)$ were aged $27-39$ years.

Conclusion: It is recommended that public health and behavioural scientists in Zimbabwe devote more time to understanding the intricacies of male sexual behaviour at different stages of life. This would provide the important insight needed to develop effective targeted interventions to reduce the spread of STDs/HIV in Zimbabwe.
\end{abstract}

\section{INTRODUCTION}

The devastation by the HIV/AIDS pandemic in subSaharan Africa is becoming increasingly apparent. The search for a cure worldwide has also proven elusive. There has therefore been a heightened sense of urgency since the past decade for behavioural research and interventions on the prevention of HIV transmission. Behavioural scientists have intensively engaged in studying and attempting to reduce high risk practices such as unprotected sexual intercourse, having multiple sexual partners and sharing of needles among intravenous drug users. The need to curb these high risk practices is clearly of paramount importance as it is presently estimated that almost 30 million HIV infections have occurred globally(1). As high as $90 \%$ of new HIV infections are forecast to occur in developing countries by the beginning of the new millennium. Women in these countries are expected to constitute the majority of all HIV/AIDS cases by then(2).

In Zimbabwe, it is presently estimated that $25.84 \%$ of the total sexually active adult population (15-49 years) is HIV positive(3). More than one thousand HIV-related deaths are reported to now occur weekly in Zimbabwe. In 1992, 25\% of all adult deaths were HIV/AIDS-related(4). According to SAFAIDS(5), persons aged 15-49 years are at increased risk due to heterosexual contact. In Southern Africa, the overall gender ratio for HIV infection is presently approximately 1:1, however, females are usually infected at younger ages than their male counterparts. This is probably because of their engagement in sexual 
relationships with older men(5). Heterosexual transmission has been identified as the dominant mode of HIV transmission in sub-Saharan Africa(6) with at least $80 \%$ of all infections attributable to this(7). Consequently, much of the AIDS research in the region has focussed on elucidating issues surrounding heterosexual HIV transmission, with particular emphasis on commercial sex workers and their clientele. However, since the HIV pandemic has made dramatic inroads into the entire sexually active population of sub-Saharan Africa, it is necessary that research on sexuality be undertaken to go beyond the highest risk groups and the highest risk contexts of commercial and casual sex. In brief, a broader understanding of sexual cultures is needed. However, sexuality is not a topic that is openly discussed in most cultures including those of sub-Saharan Africa, and there has been a paucity of research on the subject. Clarifying issues surrounding heterosexual transmission of HIV has thus proven to be somewhat difficult in the region. Nevertheless in Zimbabwe, several factors, which facilitate the spread of HIV, have been identified. Urbanisation, migrant labour, population displacement, rapid cultural changes, inadequate social services and the male-imposed inferior status of women are some of the underlying factors that exacerbate heterosexual HIV transmission(8).

In recent years, several interesting studies on HIV/ AIDS have emerged from Southern Africa, especially in Zimbabwe. These studies have not only explicated factors surrounding HIV transmission, but also indicated how male sexual behaviour could impact the risk of HIV transmission to women. Some studies have also found condom usage rate among Zimbabwean men to be low and inconsistent especially among those who had "high-risk" sexual partners $(9,10)$. Such findings highlight the plight of the African woman whose risk of contracting sexually transmitted diseases (STDs) or HIV might be increased not through her own behaviour but through that of her regular sex partner(6,11). According to Gupta and Weiss(12), Zimbabwean women are perceived by their male counterparts to have little knowledge and very limited decision making abilities on sexual matters in a relationship. The traditional gender roles in the Zimbabwean culture also limit the control women have over their sexuality. Zimbabwean women may be knowledgeable about the dangers of unprotected sex, but lack adequate empowerment to negotiate for safer sex. The social structure and belief systems which are dominated and defined by males continue to perpetuate the imbalances between both sexes. Decreasing the transmission of STDs/HIV will therefore require acknowledging and then balancing the differences in power relationships between men and women(13).

Women in Zimbabwe may also be at increased risk for STDs/HIV because of the traditional practices undertaken in an attempt to increase their partners ' sexual pleasure. Certain substances are used to decrease the amount of vaginal secretions, tighten and dry the vagina thereby raising the temperature inside the vagina. Some of these substances include absorbents, antiseptic solutions, chemical substances and herbs. Currently, there is little known about the effects of these vaginal tightening substances, but there is the possibility that the use of these substances could increasingly facilitate the transmission of STDs/HIV (14). In 1992, Runganga and co-workers(15) studied the use of herbs and other agents by women prior to sexual intercourse. The majority of these women felt that using vaginal tightening and drying agents could maintain their partner's fidelity, improve his sexual experience and sexual satisfaction. Exploring gender roles in sexuality is therefore important for the development of gender-specific HIV-preventive interventions.

This paper focusses on generational differences in Zimbabwean men's sexual behaviour that may affect their women's chances of STD/HIV acquisition. We also explored male attitudes about gender roles in sexuality.

\section{MATERIALS AND METHODS}

The sample for this study was extracted from a larger crosssectional study " The Male Sexual Behaviour and STD risk awareness study", carried out in 1996. The study assessed sexual behaviour and STD risk awareness among urban and rural Zimbabwean men aged 16 years and above. The survey for the study was carried out in two communal areas (Mashonaland and Matabeleland) and two urban areas (Harare and Bulawayo); 1496 men from these areas were randomly selected from 1000 households based on the Central Statistical Office enumeration master lists. The sample was weighted proportionally, according to the two main ecological structures of Zimbabwe (32\% urban/ peri-urban and $68 \%$ rural).

Study population: For the present study, 397 participants resident in Harare, Zimbabwe's Capital city were extracted from the overall survey sample. This sub-sample was stratified for analysis into the following age categories: 18-26 years, 27-39 years and $\geq 40$ years. These age categories were chosen to reflect different life stages. It is expected that men aged 18-26 years would still be in school or recently out of school not yet settled in employment paths and frequently not married. Men aged 2739 years would generally no longer be in school, would be working or looking for employment and for the most, be married and/or starting families. Men aged $\geq 40$ years would most likely be working, have families and a more settled life than younger men.

Data entry and statistical analysis: The data were entered into the Statistical Package for Social Sciences (SPSS)(16) data entry option software and subsequently analysed using SPSS and EPI-INFO(17) statistical softwares. The variables of interest for the hypotheses on generational differences were on: (i) sexual lifestyle behaviour that increases the risk of STD/HIV transmission to men's sexual partners; (ii) men's sexual behaviour and attitudes that affect women's abilities to protect themselves and; (iii) male attitudes about gender roles in sexuality. Our results are presented using p-values (from Chi-square tests) and/ or Odds Ratios (ORs) as indices of statistical significance. EPIINFO was used in computing the odds ratios (ORs) and their $95 \%$ confidence intervals (CIs), as appropriate for dichotomous outcome variables. 


\section{RESULTS}

The respondents were aged 18-74 years. Table 1 displays the socio-demographic characteristics of the respondents. The greatest proportion of all respondents were aged $18-26$ years $(45.1 \%)$ and belonged to a monogamous marital union $(56.1 \%)$. Majority of all respondents were educated to a secondary school level $(79.1 \%)$ and were paid employees $(58.9 \%)$ of various establishments. Most of these respondents (93.2\%) were sexually active by the time of the survey.

Table 1

Distribution of respondents according to socio-demographic characteristics

\begin{tabular}{|c|c|c|}
\hline Characteristic & No. & $\%$ \\
\hline \multicolumn{3}{|l|}{ Age(years) } \\
\hline $18-26$ & 179 & 45.1 \\
\hline $27-39$ & 119 & 30.0 \\
\hline$\geq 40$ & 98 & 24.7 \\
\hline Missing & 1 & 0.3 \\
\hline \multicolumn{3}{|l|}{ Marital status } \\
\hline Never married & 155 & 39.0 \\
\hline Married (monogamous) & 224 & 56.4 \\
\hline Married (polygamous) & 4 & 1.0 \\
\hline Cohabiting & 5 & 1.3 \\
\hline Divorced/separated/widowed & 9 & 2.3 \\
\hline \multicolumn{3}{|l|}{ Education } \\
\hline None & 4 & 1.0 \\
\hline Primary & 61 & 15.4 \\
\hline Secondary & 314 & 79.1 \\
\hline Tertiary & 18 & 4.5 \\
\hline \multicolumn{3}{|l|}{ Employment status } \\
\hline Employer & 22 & 5.5 \\
\hline Paid employee & 234 & 58.9 \\
\hline Own account worker & 17 & 4.3 \\
\hline Unpaid family worker & 4 & 1.0 \\
\hline Unemployed & 105 & 26.4 \\
\hline Student & 14 & 3.5 \\
\hline Missing & 1 & 0.3 \\
\hline \multicolumn{3}{|l|}{ Monthly income(Zimbabwe \$) } \\
\hline 0 & 71 & 17.9 \\
\hline $1-400$ & 41 & 10.3 \\
\hline $1001-2500$ & 114 & 28.7 \\
\hline $2501-5000$ & 90 & 22.7 \\
\hline$\geq 5001$ & 39 & 9.8 \\
\hline Missing & 4 & 1.0 \\
\hline
\end{tabular}

At time of survey, Zimbabwe $\$ 8.5=$ US $\$ 1$.
Males' sexual and lifestyle behaviour that increases the risk of STD/HIV transmission to their partners: Fewer men in the 27-39 year age group compared to men aged 1826 years $(22.2 \%$ versus $28.9 \%)$ reported having more than two sexual partners. Only $8.6 \%$ of the men aged 40 years and above reported having more than two sexual partners. Questions about whether condoms had ever been used were asked of participants. Significantly, fewer men in the $\geq 40$ year age group than those in the 18-26 year age group (43.8\% versus $69.5 \%$; Odds Ratio $(O R)=0.34,95 \%$ Confidence Interval (CI) $0.20-0.82$ ) had a history of condom use. When ever use of condoms was compared between men aged 27-39 years and those aged 18-26 years, there was no significant difference between proportions $(76.5 \%$ versus $69.5 \%$; OR $=1.42,95 \% \mathrm{CI}$ 0.81-2.51).

Table 2

Distribution of males' sexual behaviour that increases the risk of STDs/HIV transmission to their partners according to age.

\begin{tabular}{llll}
\hline & $\mathrm{N}(\%)$ & & \\
$18-26$ & $27-39$ & $\geq 40$ & $\chi^{2}$ \\
\hline Number of sexual partners & & & $45.59^{\mathrm{C}}$
\end{tabular}

$\begin{array}{lllllll}0 & 61 & (40.1) & 69 & (59.0) & 77 & (82.8) \\ 1 & 47 & (30.9) & 22(18.8) & 8 & (8.6) \\ \geq 2 & 44 & (28.9) & 26(22.2) & 8 & (8.6)\end{array}$

Condom use

$27.61^{\mathrm{C}}$

\begin{tabular}{llll} 
Yes & $121(69.5)$ & $91(76.5)$ & $42(43.8)$ \\
No & $53(30.5)$ & $28(23.5)$ & $54(56.3)$ \\
Odds Ratio (OR) & Referent & $1.42^{\mathrm{d}}$ & $0.34^{\mathrm{c}}$ \\
$95 \%$ CI for OR & - & $0.81-2.51$ & $0.20-0.82$ \\
\hline
\end{tabular}

a $\mathrm{p}<0.05$

b $\mathrm{p}<0.0 \mathrm{I}$

c $\mathrm{p}<0.001$

d Not significant

Males' behaviour and attitudes that affect women's abilities to protect themselves: Table 3 summarises the distribution of men according to behaviour that affect women's abilities to protect themselves according to age group. In the event that they contracted HIV, $79.5 \%$ of men aged $18-26$ years, $82.4 \%$ of those aged $27-39$ years and $85.9 \%$ of men aged $\geq 40$ years indicated that they would disclose their HIV status to their wives. Of the men with girl friends, $56.8 \%, 54.0 \%$ and $53.1 \%$ of men aged 18-26 years, 27-39 years and $\geq 40$ years respectively, indicated that they would disclose their HIV status to their girlfriends if they tested positive.

Significantly, more men in the $\geq 40$ year $(42.4 \%$; OR $=2.23,95 \%$ CI $1.19-4.18)$ and $27-39$ year $(37.5 \%$; OR = $1.82,95 \%$ CI 1.00-3.32) age groups when compared to men in the 18 -26 year age group (24.8\%) had a preference for women with dry vaginas prior to sex. 
Table 3

Distribution of males' behaviour and attitudes that affect women's abilities to protect themselves according to age.

\begin{tabular}{lllll}
\hline \multicolumn{5}{c}{$\mathrm{N}(\%)$} \\
& $18-26$ & $27-39$ & $\geq 40$ & $\chi^{2}$ \\
\hline $\begin{array}{l}\text { Likelihood of sharing HIV } \\
\text { information with wife }\end{array}$ & & & & $0.894^{\mathrm{d}}$ \\
Yes & $35(79.5)$ & $75(82.4)$ & $73(85.9)$ & \\
No & 9 & $(20.5)$ & $16(16.6)$ & $12(14.1)$ \\
Odds Ratio (OR) & Referent & $1.21^{\mathrm{d}}$ & $1.56^{\mathrm{d}}$ & \\
95\% CI for OR & - & $0.44-3.26$ & $0.54-4.46$ &
\end{tabular}

Likelihood of sharing HIV

information with girlfriend

$\begin{array}{llll}\text { Yes } & 92(56.8) & 54(54.0) & 43(53.1)\end{array}$

$\begin{array}{llll}\text { No } & 70(43.2) & 46(46.0) & 38(46.9)\end{array}$

$\begin{array}{llll}\text { Odds Ratio (OR) } \quad \text { Referent } & 0.89 \mathrm{~d} & 0.86\end{array}$

$95 \% \mathrm{CI}$ for OR $\quad$ - $\quad 0.52-1.52 \quad 0.49-1.52$

Preference for women with

dry vagina prior to sex

Yes

$\begin{array}{llll}\text { Yes } & 32(24.8) & 39(37.5) & 36(42.4) \\ \text { No } & 97(75.2) & 65(62.5) & 49(57.6) \\ \text { Odds Ratio (OR) } & \text { Referent } & 1.82^{\mathrm{a}} & 2.23^{\mathrm{b}}\end{array}$

$95 \%$ CI for OR $\quad$ - $\quad 1.00-3.32 \quad 1.19-4.18$

${ }^{\mathrm{a}} \mathrm{p}<0.05$

b $\mathrm{p}<0.01$

c $\mathrm{p}<0.00 \mathrm{I}$

d Not significant

${ }^{+}$Married respondents only

Table 4

Distribution of males' attitudes about gender role in sexuality according to age.

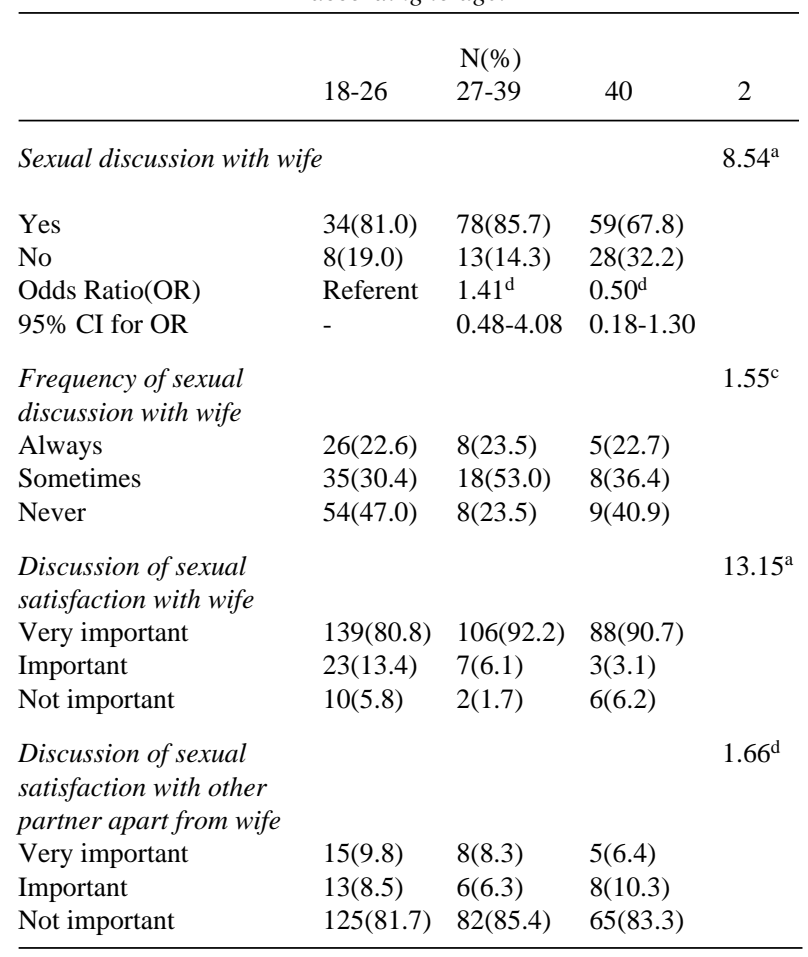

a $\mathrm{p}<0.05$

b $\quad \mathrm{p}<0.01$

$\mathrm{p}<0.001$

d Not significant

+ Married respondents only
Males' attitudes about gender roles in sexuality: Table 4 summarises the distribution of males' attitudes about gender roles in sexuality according to age group. Eighty one per cent of men in the 18-26 year age group, $85.7 \%$ of those in the $27-39$ year age group and $67.8 \%$ of those in the $\geq 40$ year age group reported that they often discussed issues relating to sexual satisfaction with their wives. However, fewer men discussed sexual satisfaction with partners other than their wives. Only $22.7 \%$ of men aged $\geq 40$ years, $23.5 \%$ of those aged $27-39$ years and $22.6 \%$ of those aged 18-26 years discussed sexual satisfaction with other partners.

A small percentage of men aged 40 years and above $(6.1 \%)$ considered sexual satisfaction with their wives important, while $1.7 \%$ and $5.7 \%$ of men aged $27-39$ years and 18-26 years respectively, also shared this belief.

\section{DISCUSSION}

The underlying assumption of this study, that there are generational differences in sexuality in relation to the spread of the HIV infection was based in part on empirical evidence of differential levels of HIV infection in different age groups in Zimbabwe. It was also based in part on the commonly held view that sexual behaviour changes as people grow older. In addition, we believe that sexual culture changes over generations. The results from this study have indicated that some generational differences in male sexual behaviour in Zimbabwe exist. According to our findings, the younger generation is initiating sexual activity during adolescence. If this sexual activity is not met with adequate interventions, they would also fall victim to the consequences of STDs/HIV. Sex education needs to begin very early in adolescence to ensure that future young adults could make decisions that would allow them to protect themselves when they do become sexually active. Given the present social disapproval of sex education for adolescents, parents may need to be targeted first in order to educate them on the merits of HIV/ AIDS knowledge for their children.

A sizeable proportion of men in each age group reported having two or more sexual partners, and no significant difference according to age was detected. Having multiple sexual partners increases the risk of contracting HIV and possibly transmitting the virus if already infected. Future interventions in the male population should highlight the importance of decreasing STD/HIV risk by avoiding multiple sexual partners.

Similar to the study of Mbizvo et al(9), a substantial proportion of younger men in this population reported having ever used condoms. This is because these men had grown up in the era of HIV/AIDS and the promotion of condom use to avoid its spread. On the other hand, older men had reached maturity in a different historical context free from HIV/AIDS. Obviously, in order to decrease the risk of contracting HIV, there must be consistency in condom use. Many of the men who reported a history of condom use in this study had multiple sexual partners. 
They only used condoms with women they had a perception of as likely to belong to a "high risk" group, for example commercial sex workers. Their regular sexual partners are hereby put at an increased risk of STD/HIV acquisition, especially if unprotected intercourse occurs with some other sexual partner(s). The fact that some men had used condoms before is however indicative that barrier protection methods are becoming increasingly accepted by Zimbabwean men. Nonetheless, additional studies are needed to examine attitudes toward condom use and frequency of condom use.

In regard to disclosing HIV status to wives or to other sexual partners, we found no significant differences according to age group. However, most married men, irrespective of age reported that they would disclose their HIV status to their wives, rather than to other sexual partners if they found out that they were HIV positive. If women eventually know of their partners HIV positive status, the issue then becomes whether or not they possess the skills to negotiate for consistent condom use. Future intervention strategies for women in Zimbabwe should incorporate education as well as condom negotiating skills. This would enable the women become equipped with the tools for successful protection against STDs/HIV especially if their partners are not faithful and/or if they are not in monogamous relationships. In addition, more female controlled protection methods are needed. Future research should also examine the acceptability of the female condom in this patriarchal society.

A reasonable percentage of men in each age group stated their preference for women with dry vaginas prior to sex. This proportion was however greatest among men aged $\geq 40$ years. Researchers have indicated that this vagina drying technique could enhance the transmission of HIV/AIDS due to small abrasions in the vaginal wall(15). Intervention measures are needed to educate men and women about the harmful effects of these drying agents.

In regard to sexual discussion with partners and the sexual satisfaction of female partners, we found no significant generational differences. Nonetheless, a reasonable proportion of men, regardless of age had a history of having sexual discussions with their wives or stable sexual partners than with other sexual partners. In other words, sexual satisfaction seems to be an issue considered more important with the wife/stable sexual partner than with other sexual partners. The results have also indicated that married men with extramarital partners usually gave their wives preferential treatment when it came to discussion of sexual satisfaction.

Despite some interesting findings, the current study is not without limitations. As noted earlier, this study is an extract of a larger study. The questionnaire was not originally designed for the type of analysis employed. Moreover, the sample only included men resident in the city of Harare, and therefore only representative of men in Harare.

\section{ACKNOWLEDGEMENTS}

Funding for this study was provided through a research support grant by the Ford Foundation to the University of Zimbabwe's Department of Obstetrics and Gynaecology. The bulk of this work was done while B.A.O was a Lecturer at the University of Zimbabwe. Special thanks to Miss L Kuwanda and Mr. M. Mawuru for being actively involved in the data collection and data entry processes.

\section{REFERENCES}

1. UNFPA. The State of the World Population, 1997.

2. Bruyn, M., Jackson, H., Wijermars, M., Knight, V.C. and Berkvens, R. Facing the challenges of HIV, AIDS and STDs: A Gender based Program Response. Royal Tropical Institute, 1995.

3. UNAIDS/WHO. Report on the Global HIV/AIDS Epidemic, June 1998.

4. City of Harare, Medical Officer of Health 1992 Annual Report, Harare, Zimbabwe, 1993.

5. Southern Africa AIDS Information Dissemination Service (SAFAIDS). The socio-economic Impact of AIDS: Issues and options, 1996.

6. DeCock, K.M., Ekpini, E., Gnaore, E., Kadio, A. and Gayle, H.D. The Public Health Implications of AIDS Research in Africa. $J$. Amer. Med. Ass. 1994; 272: 481-486.

7. Berkley, S. AIDS in the developing world: An epidemiologic overview. Clin. Infect. Dis. 1993; 17: S329-S336.

8. Jackson, H. AIDS and Social Work in Africa. J. Soc. Dev. Afr. 1991; 6: 42-62.

9. Mbizvo, M.T., Ray, S., Bassett, M., McFarland, W., Machekano, $\mathrm{R}$. and Katzenstein, D. Condom use and the risk of HIV infection who is being protected? Cent. Afr. J. Med. 1994; 40: 294-299.

10. Moyo, I.M., Ray CS, Bassett Met al. Behaviour patterns that may predispose to HIV transmission or further transmission and possible intervention strategy in the City of Harare Part II. Cent. Afr. J. Med. 1993; 39: 217-221.

11. Pitts, M., Bowman, M. and McMaster, J. Reactions to repeated STD infections: Psychosocial aspects and gender issues in Zimbabwe. Soc. Sci. Med. 1995; 40: 1299-1304.

12. Gupta, G.R. and Weiss, E. Women and AIDS: Developing a new Health Strategy. International Center for Research on Women, 1993.

13. Campbell, B. and Mbizvo, M.T. Sexual behaviour and HIV knowledge among adolescent boys in Zimbabwe. Cent. Afr. J. Med. 1994; 40: 245-250.

14. Runganga, A.O. and Kasule, J. The vaginal use of herbs/substances: An HIV facilitatory factor. AIDS Care 1995; 7: 639-645.

15. Runganga, A.O., Pitts, M. and McMaster, J. The use of herbal and other agents to enhance sexual experience. Soc. Sci. Med. 1992; 35: $1037-1042$.

16. SPSS/PC+ Version 2.0. SPSS Inc. Chicago, Illinois, 1988.

17. EPI-INFO, Version 6, A Word-Processing, Database and Statistics Program for Public Health on IBM-Compatible Microcomputers, Centre for Disease Control and Prevention (CDC), Atlanta, Georgia, 1994. 